\title{
Effect of an Automatic Triggering and Cycling System on Comfort and Patient-Ventilator Synchrony during Pressure Support Ventilation
}

\author{
Renata dos S. Vasconcelos Luíz Henrique de P. Melo Raquel P. Sales \\ Liégina S. Marinho Flávio C. Deulefeu Ricardo C. Reis \\ Mirizana Alves-de-Almeida Marcelo A. Holanda \\ Laboratory of Respiration, Department of Internal Medicine, Federal University of Ceará, Fortaleza, Brazil
}

\author{
Key Words \\ Respiratory mechanics · Respiration · Artificial · Mechanical \\ ventilation
}

\begin{abstract}
Background: The digital Auto-Trak ${ }^{\mathrm{TM}}$ system is a technology capable of automatically adjusting the triggering and cycling mechanisms during pressure support ventilation (PSV). Objective: To compare Auto-Trak with conventional settings in terms of patient-ventilator synchrony and discomfort. Methods: Twelve healthy volunteers underwent PSV via the mouth by breathing through an endotracheal tube. In the conventional setting, a pressure support of $8 \mathrm{~cm} \mathrm{H}$ with flow cycling ( $25 \%$ peak inspiratory flow) and a sensitivity of $1 \mathrm{~cm} \mathrm{H} \mathrm{H}_{2} \mathrm{O}$ was adjusted. In Auto-Trak the triggering and cycling were automatically set. Discomfort, effort of breathing, and the asynchrony index (Al) were assessed. In a complementary bench study, the inspiratory and expiratory time delays were quantified for both settings in three mechanical models: 'normal', obstructive (COPD), and restrictive (ARDS), using the ASL 5000 simulator. Results: In the volunteer study the $\mathrm{Al}$ and the discomfort scores did not differ statistically between the two settings. In the bench investigation the use of Auto-Trak was associated with a greater triggering delay in the COPD model and earlier expiratory cycling in the ARDS model but with no asynchronic events. Conclusions: Use of
\end{abstract}

the Auto-Trak system during PSV showed similar results in comparison to the conventional adjustments with respect to patient-ventilator synchrony and discomfort in simulated conditions of invasive mechanical ventilation.

Copyright $\odot 2013$ S. Karger AG, Basel

\section{Introduction}

Patient-ventilator asynchrony can be defined as a mismatch between the patient and the ventilator inspiratory and expiratory times. It is very common during assisted mechanical ventilation [1-4]. Patient-ventilator synchrony is directly related to ventilatory work [5-9]. Dyssynchrony may delay the withdrawal of ventilatory support, resulting in further complications which will potentially increase the morbidity, the mortality, and the total cost of the patient to the health system [10-12].

To minimize these problems, different approaches for optimal ventilator triggering and cycling have been developed, such as flow triggering sensitivity, adjustable flow cycling during pressure support ventilation (PSV), and 'shape signal' algorithms [12].

Digital Auto-Trak ${ }^{\mathrm{TM}}$ consists of a technology capable of automatically adjusting, breath by breath, the triggering and cycling mechanisms. Its application has the objective of reducing patient effort, improving patient comfort,

\section{KARGER}

E-Mail karger@karger.com www.karger.com/res
(C) 2013 S. Karger AG, Basel

0025-7931/13/0866-0497\$38.00/0
Renata dos Santos Vasconcelos

1120 Borges de Melo Avenue Apto 402 Fátima 60415-510 (Brazil)

E-Mail renatavasconcelos23@gmail.com 
and making assisted ventilation as close to respiratory neural activity as possible during PSV $[13,14]$. Only one study has compared the performance of Auto-Trak to customary ventilator settings in intubated patients [15].

Our main hypothesis is that the use of Auto-Trak is better in terms of synchrony and patients' sense of comfort when compared to traditional systems of triggering and cycling during PSV.

The aim of this study was to evaluate and compare the performance of an automatic triggering and cycling system, specifically Auto-Trak, with conventional pressuretriggered and flow-cycled PSV with regard to patientventilator synchrony and sense of discomfort.

\section{Methods}

This was a healthy-volunteer and also a bench study.

\section{Healthy-Volunteer Study}

This was a quantitative, interventionist, randomized, singleblind, pilot investigation. It was conducted at the Laboratory of Respiration, Department of Internal Medicine, Universidade Federal do Ceará, Brazil, from August to November, 2010.

\section{Study Subjects}

A convenience sample consisting of 12 healthy and nonsmoking volunteers over 18 years of age was recruited for this study. Volunteers suffering from diseases of the oral cavity or from dental problems were excluded.

The local institutional review board approved this study and written informed consent was obtained from each subject.

\section{Experimental Setup}

Simulated mechanical ventilation was applied through a leakproof mouthpiece device adapted to a $6.0-\mathrm{mm}$ endotracheal tube (ETT) and connected to an Esprit V-1000 mechanical ventilator (Philips Respironics ${ }^{\circledR}$, Murrysville, Penn., USA), with inspiratory and expiratory circuits. The choice of a $6.0-\mathrm{mm}$ tube was intended to simulate an increase in airway resistance as occurs during tracheal intubation [16]. The ETT was maintained in a stable position, free from kinking or flexion. An active humidification system was used. Before each experiment, the ventilator was calibrated according to the manufacturer's recommendations. Special attention was given to ensure that there were no air leaks by checking the inspiratory and expiratory tidal volumes on the ventilator screen.

The volunteer was placed in the Fowler position (inclination $45^{\circ}$ ) and the ventilator was adjusted to the corresponding setting. A period of 3-5 min before starting the experimental protocol was allowed to guarantee comfort and stabilization of the breathing pattern with positive pressure ventilation [17].

Randomization of the sequence of the two ventilation protocols was done using sealed envelopes. The order of the ventilation mode implemented depended on a sequence randomly selected for each volunteer. Each ventilation setting lasted $10 \mathrm{~min}$. A period of 5 min of washout between the protocols was allowed for the participant to rest and to minimize residual effects.
Interventional Protocols

Two protocols were tested with the ventilator on the PSV mode on room air with a pressure support of $8 \mathrm{~cm} \mathrm{H}_{2} \mathrm{O}$ and a PEEP of $4 \mathrm{~cm} \mathrm{H}_{2} \mathrm{O}$ as these are common settings during ventilatory support weaning. In the conventional setting the cycling criteria was adjusted for $25 \%$ of the peak inspiratory flow and a pressure sensitivity of $1 \mathrm{~cm} \mathrm{H} \mathrm{H}_{2} \mathrm{O}$ for triggering, since the flow triggering could generate asynchronies (auto-triggering) in the simulated conditions. There is little clinical difference between flow and pressure triggering [18]. In the Auto-Trak protocol, triggering and cycling were automatically adjusted by switching on the Auto-Trak function while the other settings were kept the same.

The Auto-Trak function applies multiple algorithms derived from flow, volume, and pressure measurements during the breath cycle to achieve its goal. There are two triggering mechanisms; whichever the ventilator detects first is used. There is the volume criterion by which triggering of the ventilator occurs when the patient's inspiratory effort drives at least $6 \mathrm{ml}$ of gas toward the ETT. The shape signal is the other mechanism, which works for both triggering and cycling of the ventilator. This is a virtual signal of the real flow curve of the previous breathing cycle, except it is 15 liters/min less and is delayed $300 \mathrm{~ms}$ in relation to the real curve. When the patient makes a respiratory effort and the real flow curve 'crosses' the virtual signal, the PS is automatically triggered. However, when the patient's inspiratory work ceases and/or expiratory effort occurs, the real flow changes to intersect the virtual signal and cycling occurs. There are also additional mechanisms of cycling, i.e. the spontaneous expiratory threshold (SET), flow reversal, and maximum inspiratory time. The SET is a method in which an electronic signal is observed in proportion to the inspiratory flow, breath by breath. When the values of the SET and the patient's effective flow are equal, the system cycles. Flow reversal is the only cycling algorithm specifically useful in cases of leaks, but in our study there were no leaks. Maximum inspiratory time is a safety feature that cycles the ventilator whenever the inspiratory time exceeds $3 \mathrm{~s}$ [13].

\section{Measurements}

In the 8 th minute of the ventilation protocol the ventilator curves (flow and pressure $\times$ time) were recorded during 2 min for offline analysis. A patient-ventilator synchrony analysis was performed via a standardized visual inspection of the pressure $\times$ time and flow $\times$ time curves by three independent observers, all pulmonologists with extensive practical experience with mechanical ventilation. The mean inspiratory time of the breathing cycles was calculated in each protocol.

The dyssynchronic events were assessed offline as previously defined by others: (1) ineffective effort, defined as an abrupt airway pressure drop $\left(\geq 0.5 \mathrm{~cm} \mathrm{H}_{2} \mathrm{O}\right)$ with a simultaneous flow reversal (in absolute value); (2) double-triggering, defined as two breath cycles separated by a very short expiratory time, i.e. less than one half of the mean inspiratory time; (3) premature cycling, defined as an inspiratory time less than one half of the mean inspiratory time, and (4) late cycling, defined as an inspiratory time greater than twice the mean inspiratory time [4]. Auto-triggering was not evaluated in the volunteer study because we did not have electromyography or esophageal pressure monitoring to ensure the absence of respiratory muscle contraction during ventilator triggering. The asynchrony index (AI) was calculated according to the method proposed by Vignaux et al. [4] using the following formula: number of dyssynchronic events/total respiratory rate $\times 100$ [19]. The 


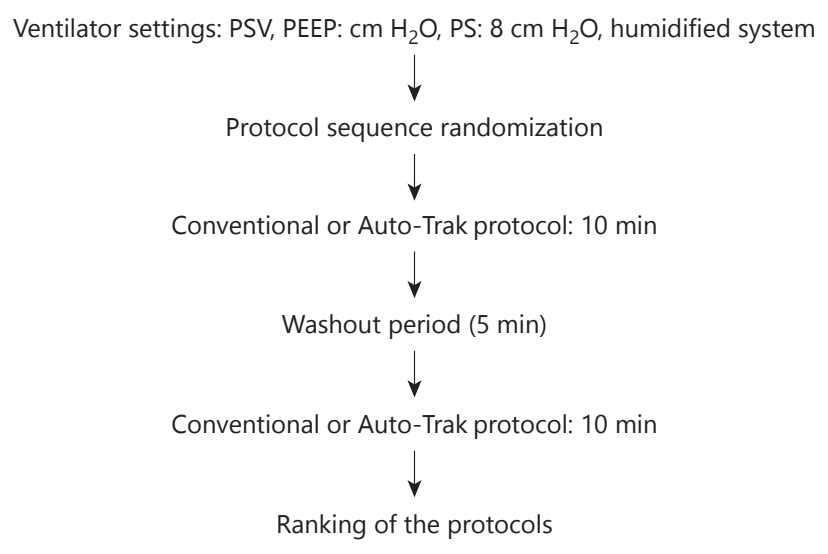

Fig. 1. Study design.

analysis for the presence of auto-PEEP was performed via a standardized visual inspection of the flow-time curve of the ventilator. Whenever the expiratory flow did not return to zero before the beginning of the next breath cycle, auto-PEEP was considered to be present in that cycle. The incidence of auto-PEEP per protocol was calculated as the number of cycles with auto-PEEP divided by the number of total cycles in 1 min multiplied by 100 .

At the end of the 10-min period of each protocol, we applied two visual analog scales (VAS), one for discomfort and the other for the sense of effort of breathing by the volunteers while the mechanical ventilation was still in use and without interference from the investigators. The VAS of discomfort consists of a straight line of $100 \mathrm{~mm}$ divided into ten segments of $10 \mathrm{~mm}$ rated from 0 to 10 , 0 being defined as no discomfort and 10 as the worst discomfort possible. The VAS for effort of breathing defined 0 as no effort and 10 as the maximum possible effort. Therefore, the lower these two scores were, the better the ventilation for the volunteer was.

At the end of the experiment the volunteers were asked to choose the best ventilation protocol according to their preference (fig. 1).

\section{Bench Study}

A bench study was conducted to refine the analysis of asynchrony, quantifying the triggering and cycling mismatch between the neural and ventilation mechanical inspiratory times and also to make possible the detection of auto-triggering and quantification of auto-PEEP, if present, and to measure the work of breathing.

Three combinations of inspiratory resistance (Rins), expiratory resistance (Rexp), and respiratory compliance (Crs) were set on the ASL 5000 simulator to simulate normal (Rins $=5 \mathrm{~cm} \mathrm{H} \mathrm{H}_{2} \mathrm{O}$ / $1 \cdot \mathrm{s}^{-1}, \operatorname{Rexp}=5 \mathrm{~cm} \mathrm{H} \mathrm{H}_{2} \mathrm{O} / \mathrm{l} \cdot \mathrm{s}^{-1}, \mathrm{Crs}=80 \mathrm{ml} / \mathrm{cm} \mathrm{H}_{2} \mathrm{O}$ ), COPD (Rins = $20 \mathrm{~cm} \mathrm{H} \mathrm{H}_{2} \mathrm{O} / \mathrm{l} \cdot \mathrm{s}^{-1}$, Rexp $=25 \mathrm{~cm} \mathrm{H} \mathrm{H}_{2} \mathrm{O} / \mathrm{l} \cdot \mathrm{s}^{-1}, \mathrm{Crs}=100 \mathrm{ml} / \mathrm{cm} \mathrm{H}_{2} \mathrm{O}$ ), and ARDS patients (Rins $=5 \mathrm{~cm} \mathrm{H}_{2} \mathrm{O} / \mathrm{l} \cdot \mathrm{s}^{-1}, \operatorname{Rexp}=5 \mathrm{~cm} \mathrm{H} \mathrm{H}_{2} \mathrm{O} / \mathrm{l} \cdot \mathrm{s}^{-1}$, $\mathrm{Crs}=30 \mathrm{ml} / \mathrm{cm} \mathrm{H}_{2} \mathrm{O}$ ) [20]. The setting of three types of respiratory mechanics was chosen to assess the influence of these variables on the triggering and cycling mechanisms. The patient's inspiratory muscle effort was configured to generate 60 liters/min of

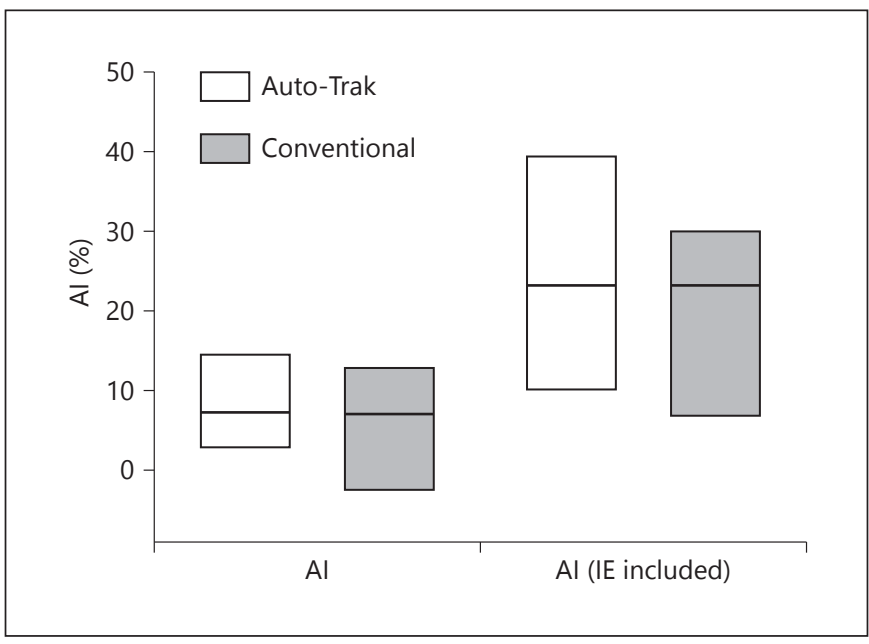

Fig. 2. Median and interquartile (25-75\%) range of the AI with and without inclusion of the IE as a type of asynchrony.

maximum inspiratory flow during spontaneous respiration in the three different patterns of respiratory mechanics [20]. The same ventilator protocols were applied in the bench study. No humidification system was used. The inspiratory rise time was set to the fastest value that did not cause an initial pressure overshoot that would shut down the mechanical lung model. In addition, the 'patient's' respiratory rate was maintained at $15 \mathrm{bpm}$. The triggering delay was defined as the difference in time from the onset of muscle effort to the beginning of the flow delivery. The cycling delay was defined as the difference between the moment of inspiratory cycling of the ventilator and the termination of 'muscle' effort. The work of breathing was calculated as $\int$ ( $\mathrm{P}$ airway - $\mathrm{P}$ chest wall) in Joules and as $\int(\mathrm{P}$ airway $-\mathrm{P}$ chest wall $) \times \mathrm{dV}$. All parameters of the bench study were determined using ASL 5000 software (Labview; National Instruments, Austin, Tex., USA).

The primary outcomes for the volunteer study were the AI, discomfort, and effort of breathing scores, and for the bench study they were triggering and cycling time delays and work of breathing.

\section{Statistical Analysis}

Statistical analysis was performed using SigmaPlot/STAT 11.0 software. The results were evaluated for normal distribution using the Kolmogorov-Smirnov normality test. If data did not present a normal distribution, the nonparametric test of Wilcoxon (signedrank test) was used for comparison between the two protocols. We only reported differences that were both statistically significant $(\mathrm{p}<0.05)$ and considered clinically important [21].

\section{Results}

\section{Volunteer Study}

The average age of the volunteers was 33.8 years (range of 21-60). Seven were female and 5 were male. No volunteer had to be excluded during the protocol. 
Fig. 3. Individual volunteer scores as measured by VAS for discomfort and effort of breathing with Auto-Trak and the conventional protocols.

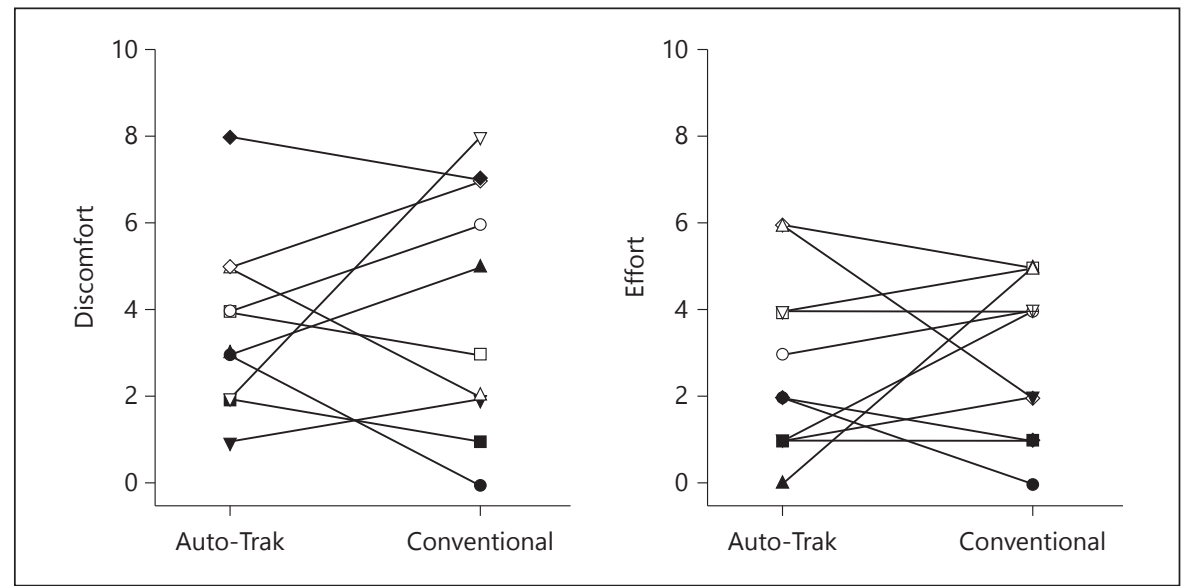

Table 1. Incidence of asynchrony events for each volunteer

\begin{tabular}{|c|c|c|c|c|c|c|}
\hline \multirow[t]{2}{*}{ Volunteer } & \multicolumn{2}{|c|}{ IEI, \% } & \multicolumn{2}{|c|}{ DTI, \% } & \multicolumn{2}{|c|}{ PCI, \% } \\
\hline & AT & Conv. & $\mathrm{AT}$ & Conv. & AT & Conv. \\
\hline 1 & 9.1 & 30 & 9.1 & 0 & 18.2 & 0 \\
\hline 2 & 11.1 & 9.1 & 0 & 0 & 0 & 9.1 \\
\hline 3 & 0 & 0 & 0 & 0 & 0 & 0 \\
\hline 4 & 0 & 0 & 0 & 0 & 0 & 0 \\
\hline 5 & 0 & 0 & 0 & 0 & 0 & 0 \\
\hline 6 & 12.5 & 15.8 & 0 & 0 & 0 & 0 \\
\hline 7 & 7.1 & 0 & 0 & 0 & 0 & 0 \\
\hline 8 & 7.1 & 8.3 & 0 & 0 & 0 & 0 \\
\hline 9 & 7.7 & 0 & 0 & 0 & 0 & 0 \\
\hline 10 & 5.9 & 5.9 & 0 & 0 & 0 & 0 \\
\hline 11 & 10 & 10 & 10 & 0 & 0 & 0 \\
\hline 12 & 16.7 & 5.5 & 0 & 0 & 0 & 0 \\
\hline Median & 7.4 & 5.7 & 0 & 0 & 0 & 0 \\
\hline Interquartile (25-75\%) & 5.2 & 8.9 & 3.7 & 0 & 5.3 & 2.6 \\
\hline
\end{tabular}

IEI = Ineffective effort index; DTI = double triggering index; $\mathrm{PCI}=$ premature cycling index; $\mathrm{SD}=$ standard deviation; Conv. = conventional system.

Figure 2 shows the Auto-Trak and the conventional mode (pressure triggered) AI. The differences were not statistically significant ( $\mathrm{p}=0.313$ for the AI).

The distribution of the types of the dyssynchronic events found in the Auto-Trak and conventional protocols for each individual is shown in table 1.

There were no statistically significant differences in the scores for discomfort and respiratory effort as measured by VAS between the two protocols. Auto-Trak had average scores of $3.8 \pm 1.9$ and $2.5 \pm 2.1$, respectively, and the conventional settings had scores of $4.3 \pm 2.7$ and $3.2 \pm$ 1.9 , respectively (figure 3 ).
Table 2. Median and interquartile (25-75\%) range of the tidal volume (TV), inspiratory time (IT), respiratory rate (RR), and percentage of breathing cycles with auto-PEEP in the Auto-Trak and conventional protocols

\begin{tabular}{|c|c|c|c|}
\hline & Auto-Trak & Conventional & $\mathrm{p}$ value \\
\hline $\mathrm{TV}, \mathrm{ml}$ & & & 0.791 \\
\hline Median & 1,018 & 889 & \\
\hline \multicolumn{4}{|c|}{ Interquartile (25-75\%) } \\
\hline range & $698-1,209$ & $642-1,358$ & \\
\hline Inspiratory time, $s$ & & & 0.084 \\
\hline Median & 2.0 & 2.2 & \\
\hline \multicolumn{4}{|l|}{ Interquartile (25-75\%) } \\
\hline $\mathrm{RR}, \mathrm{bpm}$ & & & 0.072 \\
\hline Median & 14 & 12 & \\
\hline \multicolumn{4}{|l|}{ Interquartile (25-75\%) } \\
\hline range & $11-15$ & $11-14$ & \\
\hline Auto-PEEP (\%) & & & 0.954 \\
\hline Median & 0.7 & 0.7 & \\
\hline \multicolumn{4}{|l|}{ Interquartile (25-75\%) } \\
\hline range & $0.7-0.96$ & $0.47-1.0$ & \\
\hline
\end{tabular}

Table 2 shows the median and interquartile (25-75\%) range for the tidal volume, inspiratory time, respiratory rate, and percentage of cycles with auto-PEEP in both protocols. No significant differences were observed. There was no statistically significant difference in relation to the percentage of respiratory cycles with auto-PEEP present between the Auto-Trak and conventional systems $(\mathrm{p}=0.954)$.

Seven volunteers (58.4\%) chose Auto-Trak as the best ventilation protocol and $5(41.6 \%)$ preferred the conventional mode. 


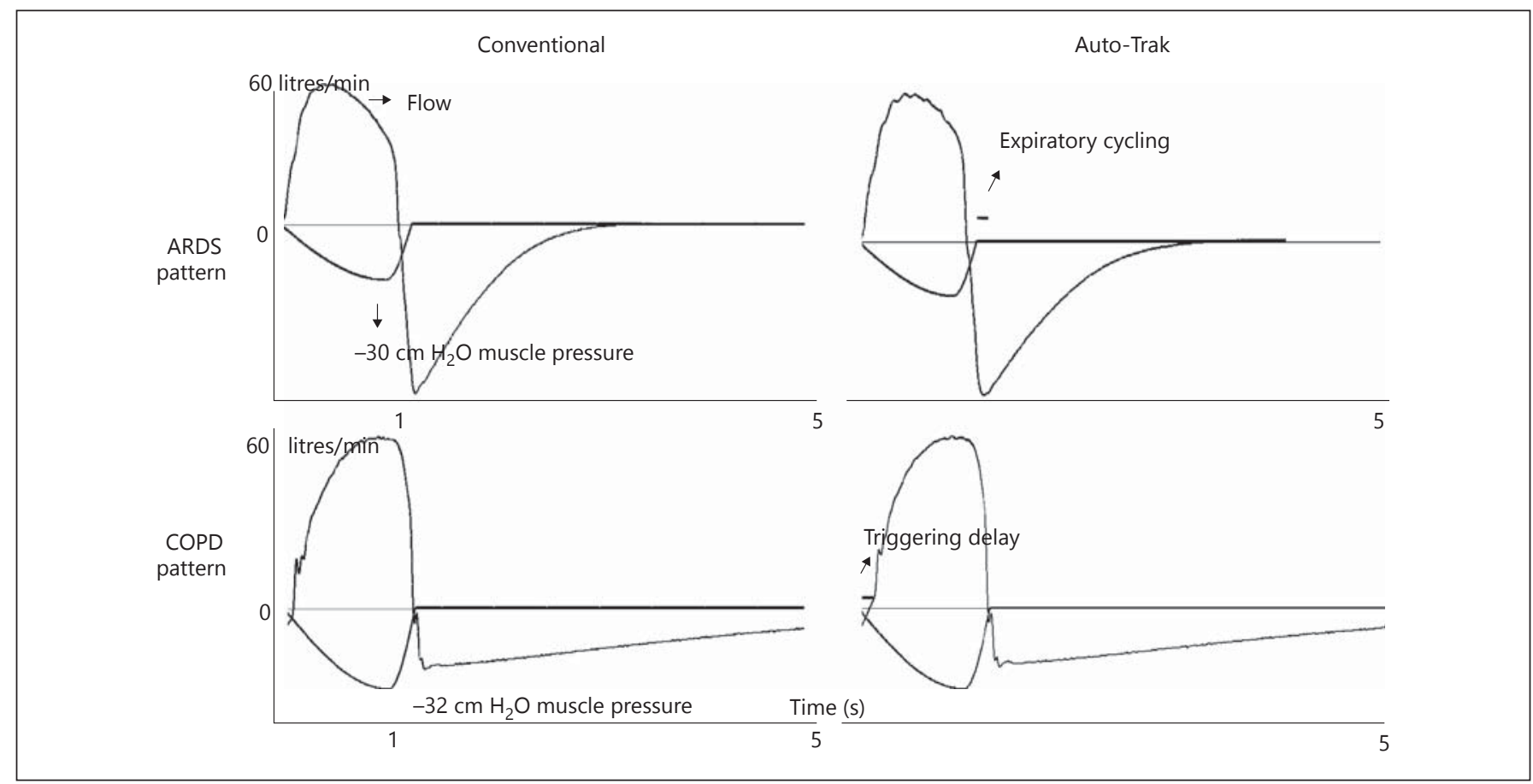

Fig. 4. Representative breathing cycles of the restrictive pattern (ARDS) and the obstructive pattern (COPD) during the conventional and Auto-Trak protocols.

Table 3. Ventilation and synchronization variables in the three patient models [normal, restrictive (ARDS), and obstructive (COPD)] with conventional and Auto-Trak protocols

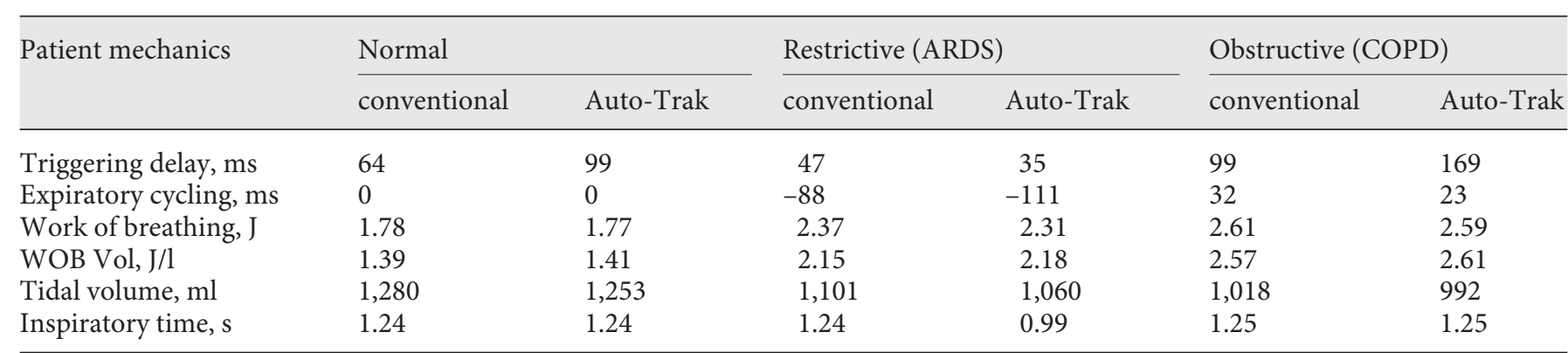

\section{Bench Study}

Table 3 shows the results for the bench study. AutoTrak showed a better performance for triggering but not for cycling the ventilator in the ARDS model. The conventional mode performed better in triggering the ventilator in the obstructive 'patient'. These differences were small $(<100 \mathrm{~ms})$. No significant differences were noted concerning the work of breathing between the protocols.

Figure 4 shows representative tracings of the ARDS and the COPD patterns during conventional and AutoTrak protocols.

Effect of an Automatic Triggering and Cycling System during PSV

\section{Discussion}

In this pilot study, which simulated invasive mechanical ventilation, use of the Auto-Trak system presented similar results in comparison to the conventional mode of pressure triggering and flow cycling with respect to patient-ventilator synchrony and sense of discomfort during PSV. The bench study showed that triggering and cycling delays were marginally affected by the Auto-Trak system in different ways according to the respiratory mechanics. 
The Auto-Trak technology was developed initially for noninvasive ventilation (NIV) equipment, and it was subsequently implemented in ICU ventilators [15]. To our knowledge, this is the first study to evaluate the AutoTrak system via an ETT and using an ICU ventilator in comparison to the conventional settings. Auto-Trak performed better only for the triggering phase in the ARDS pattern while the conventional setting was better in the triggering phase for the obstructive pattern and in the cycling phase for the ARDS pattern, but the differences were small.

Others investigators have compared flow and flow waveform triggering methods with the same Auto-Trak technology in intubated patients but with an NIV ventilator [15]. The flow waveform triggering was more sensitive to patient effort than was the flow triggering, resulting in fewer episodes of ineffective efforts. However, it resulted in a greater number of auto-triggerings, something which was not seen in our work, at least in the bench study. The authors concluded that the flow waveform system may become unstable under certain circumstances, which can have serious consequences for the patient-ventilator interaction $[15,22]$. That may be the case during NIV where air leaks are unavoidable, but not during invasive ventilation with no leaks. In fact, we did not find any type of increased instability or asynchronies during mechanical ventilation with the Auto-Trak system.

Besides the defined types of asynchronies in the literature, we also evaluated the incidence of inspiratory airway pressure excess. When considering this phenomenon as an additional type of asynchrony, the AI increased significantly. Although its clinical significance is unknown, its presence was more frequent than the other asynchronies, pointing to the fact that a more refined analysis of the patient-ventilator interaction could be conducted in the future.

Studies have shown that an average triggering delay between 100 and $120 \mathrm{~ms}$ does not generate important clinical effects $[23,24]$. In the bench study, the Auto-Trak system was associated with a triggering delay higher than this threshold only in the presence of increased airway resistance (COPD pattern). In fact, the triggering delay was $70 \mathrm{~ms}$ longer than the conventional settings. The clinical significance of this small difference is unknown.

The present study has several strengths and limitations. The study used multiple simulation techniques to assess the performance of PSV triggering and cycling algorithms. We used a $6.0-\mathrm{mm}$ and not an $8.0-\mathrm{mm}$ ETT as previous investigators have shown that the in vivo performance of this device may be comparable to that of new tubes 1-4 sizes smaller [16]. The experimental model was able to induce the appearance of asynchronies in healthy volunteers, especially ineffective effort and double triggering. Ineffective efforts may be associated with less obvious changes in flow tracing when analyzed by respiratory polygraphy, and the incidence of this type of asynchrony could have been underestimated. Thille et al. [1] studied 62 intubated patients who used mechanical ventilation with pressure support for more than $24 \mathrm{~h}$, finding a high AI ( $\geq 10 \%)$ in $24 \%$ of them. The ineffective effort and double triggering types of asynchrony accounted for more than $98 \%$ of the total number of events. These two types of asynchrony are most common in patients undergoing invasive mechanical ventilation, representing at least $80 \%$ of asynchronous breaths [9]. In the present investigation, healthy volunteers and simulated mechanical conditions and not real patients were evaluated. Therefore, comparisons of our results with those obtained in clinical studies should be made with caution. The results of this study certainly require confirmation in clinical trials involving ICU patients. In the healthy-volunteer study we used a mouthpiece device adapted to a tracheal tube and not tracheal intubation for obvious ethical reasons, which might have influenced the sense of discomfort in volunteers as well as the performance of the ventilator. The mechanical ventilation period was only $10 \mathrm{~min}$ and a different number of asynchronies could have occurred if the period of observation had been longer. Only one type of ICU mechanical ventilator was used, and the AI may be influenced by the variable performance of different ventilators. On the other hand, this fact enabled us to evaluate Auto-Trak without confounding factors related to technological differences among ventilators.

The practical implication of this study is reinforcement of the importance of optimization of ventilator triggering and cycling settings during PSV, since asynchronies are a common phenomenon even when precautions are taken. Systems that automatically perform this optimization can be useful. Auto-Trak, for example, may be more practical in comparison to conventional settings because it is not necessary to adjust triggering and cycling thresholds, simplifying the management of the ventilator by the operator. This may be particularly important when the physician involved in the care of the patient is not an expert in the field of mechanical ventilation [25].

In conclusion, use of the Auto-Trak system of automatic triggering and cycling during PSV presented results similar to the conventional settings with pressure triggering and flow cycling during PSV with respect to patientventilator synchrony and discomfort. 


\section{References}

$>1$ Thille AW, Rodriguez P, Cabello B, Lellouche F, Brochard L: Patient-ventilator asynchrony during assisted mechanical ventilation. Intensive Care Med 2006;32:1515-1522.

$>2$ Mellott KG, Grap MJ, Munro CL, Sessler CN, Wetzel PA: Patient-ventilator dyssynchrony: clinical significance and implications for practice. Crit Care Nurse 2009;29:41-55.

$\checkmark 3$ Chao DC, Scheinhorn DJ, Stearn-Hassenpflug M: Patient-ventilator trigger asynchrony in prolonged mechanical ventilation. Chest 1997;112:1592-1599.

4 Vignaux L, Vargas F, Roeseler J, Tassaux D, Thille AW, Kossowsky MP, Brochard L, Jolliet P: Patient-ventilator asynchrony during non-invasive ventilation for acute respiratory failure: a multicenter study. Intensive Care Med 2009;35:840-846.

$\checkmark 5$ Vitacca M, Bianchi L, Zanotti E, Vianello A, Barbano L, Porta R, Clini E: Assessment of physiologic variables and subjective comfort under different levels of pressure support ventilation. Chest 2004;3:851-859.

-6 Nilsestuen JO, Hargett KD: Using ventilator graphics to identify patient-ventilator asynchrony. Respir Care 2005;50:202-234.

7 Tobin MJ, Jubran A, Laghi F: Patient-ventilator interaction. Am J Respir Crit Care Med 2001;163:1059-1063.

$>8$ Mulqueeny Q, Ceriana P, Carlucci A, Fanfulla F, Delmastro M, Nava S: Automatic detection of ineffective triggering and double triggering during mechanical ventilation. Intensive Care Med 2007;33:20142018.
-9 De Wit M: Monitoring of patient-ventilator interaction at the bedside. Respir Care 2011; 56:61-72.

10 Jolliet P, Tassaux D: Clinical review: patientventilator interaction in chronic obstructive pulmonary disease. Crit Care 2006;10:236241.

11 Kondili E, Prinianakis G, Georgopoulos D: Patient-ventilator interaction. Br J Anaesth 2003;91:106-119.

12 Oakes DF, Shortall SP: Obtaining Optimal Patient-Ventilator Synchrony. Coral Springs, Respiratory Books, a division of Health Educator Publications, 2005.

13 Respironics Inc: Digital Auto-Trak. Pitts burgh, Respironics, 2000.

14 Respironics Inc: Operator's Manual Esprit Ventilator $^{\circledR}$ - Auto-Trak Sensitivity ${ }^{\mathrm{TM}}$. Murrysville, Respironics, 2005.

15 Kondili E, Prinianakis G, Georgopoulos D: Effects of the flow waveform method of triggering and cycling on patient-ventilator interaction during pressure support. Intensive Care Med 2003;29:1950-1959.

16 Wilson AM, Gray DM, Thomas JG: Increases in endotracheal tube resistance are unpredictable relative to duration of intubation. Chest 2009;136:1006-1013.

17 Laghi F, Karamchandani K, Tobin MJ: Influence of ventilator settings in determining respiratory frequency during mechanical ventilation. Am J Respir Crit Care Med 1999;160: 1766-1770.
8 Tütüncü AS, Cakar N, Camci E, Esen F, Telci L, Akpir K: Comparison of pressure- and flow-triggered pressure-support ventilation on weaning parameters in patients recovering from acute respiratory failure. Crit Care Med 1997;25:756-760.

19 Hess DR: Patient-ventilation interaction during noninvasive ventilation. Respir Care 2011;56:153-167.

20 Ferreira JC, Chipman DW, Kacmarek RM: Trigger performance of mid-level ICU mechanical ventilators during assisted ventilation: a bench study. Intensive Care Med 2008; 34:1669-1675.

-21 Ferreira JC, Chipman DW, Hill NS, Kacmarek RM: Bilevel vs. ICU ventilators providing noninvasive ventilation: effect of system leaks: a COPD lung model comparison. Chest 2009;136:448-456.

22 Georgopoulos D, Kondili E, Prinianakis G: Bedside waveforms interpretation as a tool to identify patient-ventilator asynchronies. Intensive Care Med 2006;32:34-47.

23 Kondili E, Xirouchaki N, Georgopoulos D: Modulation and treatment of patient-ventilator dyssynchrony. Curr Opin Crit Care 2007; 13:84-89.

24 Vignaux L, Tassaux D, Jolliet P: Performance of noninvasive ventilation modes on ICU ventilators during pressure support: a bench model study. Intensive Care Med 2007;33:1444-1451.

25 Uzawa Y, Yamada Y, Suzukawa M: Evaluation of the user interface simplicity in the modern generation of mechanical ventilators. Respir Care 2008;53:329-337. 